\title{
Dynamic Analysis of a Deeply Buried Tunnel Influenced by a Newly-built Adjacent Cavity with a Special Emphasis on the Minimum Seismically Safe Tunnel Distance
}

\author{
Elefterija Zlatanovićí ${ }^{1 *}$ Dragan Č. Lukić ${ }^{2}$, Vlatko Šešov $^{3}$, Zoran Bonić $^{1}$ \\ ${ }^{1}$ University of Niš, Faculty of Civil Engineering and Architecture, Aleksandra Medvedeva 14, 18000 Niš, Republic of Serbia \\ ${ }^{2}$ University of Novi Sad, Faculty of Civil Engineering of Subotica, Kozaračka 2a, 24000 Subotica, Republic of Serbia \\ ${ }^{3}$ University "Ss. Cyril and Methodius" of Skopje, Institute of Earthquake Engineering and Engineering Seismology, Todor Aleksandrov \\ 165, 1000 Skopje, Republic of North Macedonia \\ * Corresponding author, e-mail: elefterija.zlatanovic@gaf.ni.ac.rs
}

Received: 26 July 2021, Accepted: 28 September 2021, Published online: 04 October 2021

\begin{abstract}
Contemporary life streams, more often than ever, impose the necessity for construction of new underground structures in the vicinity of existing tunnels, with an aim to accommodate transportation systems and utility networks. A previously uninvestigated case, in which a newly-constructed tunnel opening is closely positioned behind an existing tunnel, referred to as the tunnel-cavity configuration, has been considered in this study. An exact analytical solution is derived considering a pair of parallel circular cylindrical structures of infinite length, with the horizontal alignment, embedded in a boundless homogeneous, isotropic, elastic medium and excited by timeharmonic plane SV-waves under the plane-strain conditions. The Helmholtz decomposition theorem, the wave functions expansion method, the translational addition theorem for bi-cylindrical coordinates, and the pertinent boundary conditions are jointly employed in order to develop a closed-form solution of the corresponding boundary value problem. The primary goal of the present study is to examine the increase in dynamic stresses at an existing tunnel structure due to the presence of a closely driven unlined cavity, as well as in a localized region around the tunnel (at the position of the cavity in close proximity), under incident SV-waves. A new quantity called dynamic stress alteration factor is introduced and the aspect of the minimum seismically safe distance between the two structures is particularly considered.
\end{abstract}

\section{Keywords}

tunnel-cavity configuration, incident SV-wave, exact solution, dynamic stress alteration factor, minimum seismically safe spacing

\section{Introduction}

For the reasons of the overpopulation, lack of space, and rising density of transportation, nowadays, tunnels have a significant role in the development of urban areas. Transportation networks, with tunnels as their integral parts, are considered to be of paramount importance when the risk under strong earthquakes is considered [1], since the accessibility of roads affects the speed and scope of emergency measures to be provided in very immediate post-earthquake emergency and relief operations, and since the earthquake-induced damage to infrastructure could severely affect the economy of a region due to the time required to restore the functionality of the network.

Recently, two smaller one-way tunnels have usually been built, which have numerous advantages, such as the reduction of both the tunnel diameter and the soil movement resulted from the construction of the tunnel. Furthermore, the construction of a third tunnel between two main structures is also becoming a practice, to provide protection in the event of accidental fire incidents or to connect the main tunnels located deep underground with the ground level [2]. Moreover, there are cases of even four tunnels in close proximity, among which the most famous is the Wushaoling Tunnel as the longest tunnel in China at present with the length of $22 \mathrm{~km}$ [3], as well as the four closely running subway tunnels in Kyoto City in Japan [4]. In many cases, for the need of accommodation of transportation systems and utility networks, a new tunnel has to be constructed closely located to an existing one. The construction of an adjacent tunnel opening will certainly affect the design ground motion along 
the cavity, and further affect seismic safety of the existing underground structure nearby. Therefore, its effect on the seismic performance of the existing tunnel structure in close proximity is quite significant and interaction mechanisms are highly complex, as the calculation should be based on an analysis of the interaction of the entire complex of structures with the surrounding medium [5].

Considering that quite often tunnels are located under densely populated urban areas, these structures require very high standards in terms of their stability and safety. Nevertheless, even in the most developed industrial countries, a perceptible discrepancy between the presently relevant provisions for underground structures and the requirements for design and construction of safe and cost-efficient underground facilities is observable nowadays, whereas the efficiency in terms of systematic and precisely established design and construction rules from the seismic point of view is even poorer [6]. The worst scenarios related to the seismic failure of two tunnels in close proximity impose the need for deeper consideration in terms of the design and construction of these structures, in particular given the fact that investigations of the mutual effect of closely spaced structures are still in the preliminary stage. The case of closely running tunnel structures should be turned into an important direction of the further development of seismic design codes, where the aspect of their minimum seismically safe distance should be a matter of concern.

Analytical studies of the seismic response of a pair of embedded structures, starting from the $60 \mathrm{~s}$ and $70 \mathrm{~s}$ of the last century, were related to the problem of P-, SV-, and SH-waves diffraction by two unlined cavities or two lined tunnels, in which the surrounding ground was considered as an infinite or a semi-infinite elastic medium [7-16]. The problem involving the multiple scattering of seismic waves by a pair of cavities or tunnels by treating the soil medium poroelastic has recently been intensively investigated [17-20]. It is evident that the literature deals with the seismic wave scattering involving twin cavities, as well as twin tunnels, considering both the case of an elastic (single-phase) medium and the case of a poroelastic (multiphase) medium, whereas it seems that to date the dynamic problems that accompany the case of a new unlined tunnel under construction near a lined tunnel in operation have not been investigated extensively.

Considering all the former facts, in the focus of this study is the dynamic response of a system comprised of an existing tunnel (lined structure) in the vicinity of which an unlined circular tunnel (tunnel opening, cavity) arises (i.e., the tunnel-cavity system). For that purpose, an exact analytical solution was derived, based on which an increase of seismically induced stresses in the existing tunnel lining and in the medium surrounding the tunnel due to the presence of a new adjacent tunnel opening is investigated, considering the effects of incident SV-wave frequencies and the distance between these two structures. The results obtained by this study are considered valuable for establishing design rules, whereby the aspect of the minimum seismically safe distance of the newly constructed tunnel opening in the vicinity of the existing tunnel was of a special importance. To date, research has mainly focused on determining the critical center-to-center distance between a new bored tunnel and an existing one from the static (construction) aspect ( $2 D-3 D, D$ being a tunnel diameter [21-23]). So that, another important goal of this research was to examine whether the statically assessed values of the minimum tunnel distance are appropriate from the dynamic aspect as well.

\section{Analytical solution}

The problem geometry is depicted in Fig. 1. A circular tunnel with the center at the origin $\mathrm{O}_{1}$ of the cylindrical coordinate system $\left(r_{1}, \theta_{1}\right)$, external radius $b_{1}$, and internal radius $a_{1}$ and a circular cavity with the center at the origin $\mathrm{O}_{2}$ of the cylindrical system $\left(r_{2}, \theta_{2}\right)$ and radius $b_{2}$ are parallel linear circular cylinders of infinite lengths and of the same size $\left(b_{1}=b_{2}\right)$, and placed side by side at a center-to-center distance $d$. The structures are deeply embedded, thus the effect of the ground surface is neglected. The rock material surrounding the tunnel and the cavity is considered as a boundless homogeneous, isotropic, elastic medium with material properties presented in terms of Lame's constants $\lambda_{\text {med }}$ and $\mu_{\text {med }}$ and mass density $\rho_{\text {med }}$. The tunnel lining is also assumed to behave in the linear elastic manner, and analogously, its material properties are $\lambda_{\text {lin }}$, $\mu_{l i n}$, and $\rho_{l i n}$. It is considered that the transverse response is separated from the longitudinal response (assuming

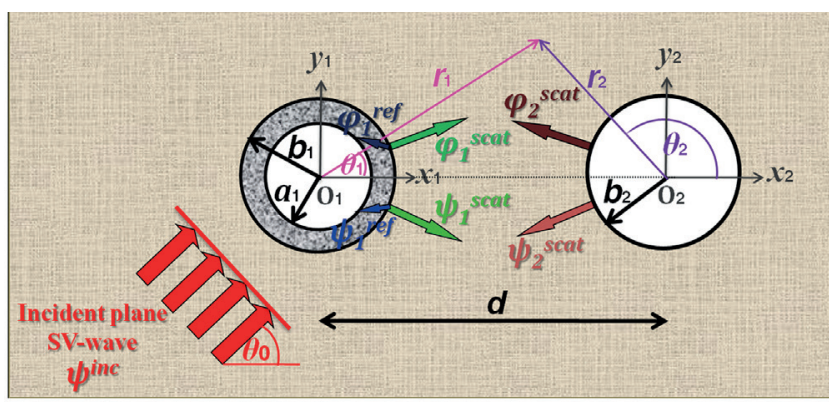

Fig. 1 Geometry of the tunnel-cavity system 
uniform properties of the tunnel lining and rock mass along the lengths of the tunnels), with the direction of wave propagation in planes perpendicular to the longitudinal axes of the structures (two-dimensional 2D planestrain conditions). The propagation of incident seismic plane SV-waves with an in-plane incidence angle $\theta_{0}$ relative to the horizontal is considered. The multiple scattering of waves between the tunnel and the cavity, as well as the waves refracted into the tunnel lining, are fully taken into account in the formulation. The construction effects for the newly built tunnel opening are disregarded.

For a homogeneous, isotropic, and elastic medium, the displacement vector components $\boldsymbol{u}_{\text {med }}$ are presented by a following system of partial differential equations [24]:

$$
\left(\lambda_{\text {med }}+\mu_{\text {med }}\right) \nabla \nabla \cdot \boldsymbol{u}_{\text {med }}+\mu_{\text {med }} \nabla^{2} \boldsymbol{u}_{\text {med }}=\rho_{\text {med }} \ddot{\boldsymbol{u}}_{\text {med }},
$$

in which $\nabla$ is the vector differential operator (nabla), $\nabla^{2}$ is the Laplacian, and dots over a quantity $\boldsymbol{u}_{\text {med }}$ mean its partial derivative with respect to time.

Considering the plane-strain nature of the problem, by applying the Helmholtz decomposition theorem (according to which the displacement field $\boldsymbol{u}_{\text {med }}$ can be represented as a superposition of longitudinal and transverse vector components), for the time-harmonic plane incident SV-wave, Eq. (1) are reduced to decoupled Helmholtz equations for a steady-state response, satisfied by the scalar potentials $\varphi_{\text {med }}$, related to the longitudinal $\mathrm{P}$-wave, and $\psi_{\text {med }}$, related to the vertically polarized shear (SV-) wave, which propagate through the medium:

$$
\begin{aligned}
& \nabla^{2} \varphi_{\text {med }}+k_{P_{\text {med }}}^{2} \varphi_{\text {med }}=0, \\
& \nabla^{2} \psi_{\text {med }}+k_{S_{\text {med }}}^{2} \psi_{\text {med }}=0 .
\end{aligned}
$$

In these expressions, $k_{P m e d}$ and $k_{\text {Smed }}$ are wavenumbers of the compressional P-waves and the shear SV-waves in the medium, respectively, which are the functions of the circular frequency of the input motion $\omega$ and the relevant wave propagation velocity in the elastic medium $c_{\text {med }}$ depending on the properties of the medium:

$$
\begin{aligned}
& k_{P_{\text {med }}}=\frac{\omega}{c_{P_{\text {med }}}}, \quad k_{S_{\text {med }}}=\frac{\omega}{c_{S_{\text {med }}}}, \\
& c_{P_{\text {med }}}=\sqrt{\frac{\lambda_{\text {med }}+2 \mu_{\text {med }}}{\rho_{\text {med }}}} \cdot c_{S_{\text {med }}}=\sqrt{\frac{\mu_{\text {med }}}{\rho_{\text {med }}}} .
\end{aligned}
$$

Equations (1)-(5) also refer to the elastic tunnel lining with the replacement of the subscript "med" with "lin".
The method of wave function expansion [25] is applied to obtain the series solution to Eqs. (2) and (3).

In determining the frequency equation, the expansion of the incident plane $S V$-wave of potential amplitude $\psi_{0}$ equal to unity, for the cylindrical wave functions in the coordinate system of each cylinder, is given as:

$\psi_{i}^{i n c}\left(r_{i}, \theta_{i}, \omega\right)=\sum_{n=-\infty}^{\infty} \vartheta_{i n} J_{n}\left(k_{S_{\text {med }}} r_{i}\right) \mathrm{e}^{\mathrm{i} n \theta_{i}}$.

$J_{n}$ is the cylindrical Bessel function of the first kind of integer order $n$, which simulates incoming waves propagating towards the cylinders, $\mathrm{i}=\sqrt{-1}$ is the imaginary unit, $i=1,2$ denotes the corresponding coordinate system (1 related to the tunnel, 2 to cavity), $\vartheta_{1 n}=\mathrm{i}^{n} \exp \left(-\mathrm{i} n \theta_{0}\right)$, $\vartheta_{2 n}=\vartheta_{1 n} \exp \left(\mathrm{i} k_{S_{\text {med }}} d \cos \theta_{0}\right)$.

In general, it should be anticipated that a transverse SV-wave, incident on the tunnel-cavity system, will give rise to scattering (reflection) of SV-waves ( $\psi_{1}^{\text {scat }}$ by the tunnel and $\psi_{2}^{\text {scat }}$ by the cavity), but also scattering (reflection) of longitudinal P-waves ( $\varphi_{1}^{\text {scat }}$ by the tunnel and $\varphi_{2}^{\text {scat }}$ by the cavity). Hence, the corresponding scattered wave potentials from the origin (center) of each structure, represented in terms of infinite Fourier-Bessel series, are:

$$
\begin{aligned}
& \varphi_{i}^{\text {scat }}\left(r_{i}, \theta_{i}, \omega\right)=\sum_{n=-\infty}^{\infty} A_{\text {in }}(\omega) H_{n}^{(1)}\left(k_{P_{\text {med }}} r_{i}\right) \mathrm{e}^{\mathrm{i} n \theta_{i}}, \\
& \psi_{i}^{\text {scat }}\left(r_{i}, \theta_{i}, \omega\right)=\sum_{n=-\infty}^{\infty} B_{\text {in }}(\omega) H_{n}^{(1)}\left(k_{S_{\text {med }}} r_{i}\right) \mathrm{e}^{\mathrm{i} n \theta_{i}} .
\end{aligned}
$$

Here, $H_{n}^{(1)}$ is the cylindrical Hankel function of the first kind of integer order $n$ and it represents outgoing waves that propagate away from the cylinders. $A_{i n}(\omega)$ and $B_{\text {in }}(\omega)$ are unknown scattering coefficients.

The total wave field potentials in the elastic medium in the presence of the tunnel-cavity system are comprised of the incident wave field and the scattered wave field $\left(\varphi_{\text {med }}=\varphi_{i}^{\text {scat }}+\varphi_{j}^{\text {scat }}\right.$ and $\psi_{\text {med }}=\psi_{i}^{\text {inc }}+\psi_{i}^{\text {scat }}+\psi_{j}^{\text {scat }}$ for $i, j=1,2(i \neq j))$. Before applying the boundary conditions, the scattered field from the $j$-th structure, expressed in the $j$-th coordinate system, has to be transformed to the coordinate of the $i$-th structure (in this way, the dynamic interaction between the tunnel and the cavity is fully considered) by the aid of the translational addition theorem [26]:

$H_{n}^{(1)}\left(k_{\text {med }} r_{i}\right) \mathrm{e}^{\mathrm{i} n \theta_{i}}=$

$\sum_{m=-\infty}^{\infty} H_{n-m}^{(1)}\left(k_{m e d} d\right) J_{m}\left(k_{m e d} r_{j}\right) \mathrm{e}^{\mathrm{i}(n-m) \theta_{i j}} \mathrm{e}^{\mathrm{i} m \theta_{j}}$, 
which applies both to the compressional wavenumber $\left(k_{\text {Pmed }}\right)$ and the shear wavenumber $\left(k_{\text {Smed }}\right)$ and in which $\theta_{i j}$ is an angle between the $\mathrm{O} j \mathrm{O} i$ line and the $x_{i}$-axis. Thus, the total wave field potentials with respect to only one coordinate system (the coordinate system of the $i$-th cylinder) will be:

$$
\begin{aligned}
& \varphi_{\text {med }}\left(r_{i}, \theta_{i}, \omega\right)=\sum_{n=-\infty}^{\infty} A_{i n}(\omega) H_{n}^{(1)}\left(k_{P_{\text {med }}} r_{i}\right) \mathrm{e}^{\mathrm{i} n \theta_{i}}+ \\
& +\sum_{n=-\infty}^{\infty} \sum_{m=-\infty}^{\infty} A_{j m}(\omega) H_{m-n}^{(1)}\left(k_{P_{\text {med }}} d\right) J_{n}\left(k_{P_{\text {med }}} r_{i}\right) \mathrm{e}^{\mathrm{i}(m-n) \theta_{j i}} \mathrm{e}^{\mathrm{i} n \theta_{i}}, \\
& \psi_{\text {med }}\left(r_{i}, \theta_{i}, \omega\right)= \\
& =\sum_{n=-\infty}^{\infty}\left[\vartheta_{i n} J_{n}\left(k_{S_{\text {med }}} r_{i}\right)+B_{\text {in }}(\omega) H_{n}^{(1)}\left(k_{S_{\text {med }}} r_{i}\right)\right] \mathrm{e}^{\mathrm{i} n \theta_{i}}+ \\
& +\sum_{n=-\infty}^{\infty} \sum_{m=-\infty}^{\infty} B_{j m}(\omega) H_{m-n}^{(1)}\left(k_{S_{\text {med }}} d\right) J_{n}\left(k_{S_{\text {med }}} r_{i}\right) \mathrm{e}^{\mathrm{i}(m-n) \theta_{j i}} \mathrm{e}^{\mathrm{i} n \theta_{i}},
\end{aligned}
$$

for $i, j=1,2(i \neq j)$. From Fig. 1 , it can be seen that for the considered horizontally aligned tunnel-cavity system, with a common $x$-axis, the angle $\theta_{12}$ is equal to 0 and the angle $\theta_{21}$ is equal to $\pi\left(180^{\circ}\right)$.

When considering the lining of the existing tunnel, in addition to the two reflected waves, which arise from the incident SV-wave arriving at the rock-lining interface, two waves, a P-wave and an SV-wave, refracted through the elastic tunnel liner also arise. These waves are considered as standing waves, reflected back and forth between the two surfaces of the lining. The relevant scalar potentials of the refracted waves in the elastic tunnel lining are:

$\varphi_{\text {lin }}=\varphi_{1}^{r e f}\left(r_{1}, \theta_{1}, \omega\right)=$

$\sum_{n=-\infty}^{\infty}\left[C_{1 n}(\omega) J_{n}\left(k_{P_{l i n}} r_{1}\right)+D_{1 n}(\omega) Y_{n}\left(k_{P_{l i n}} r_{1}\right)\right] \mathrm{e}^{\mathrm{i} n \theta_{1}}$,

$\psi_{\text {lin }}=\psi_{1}^{r e f}\left(r_{1}, \theta_{1}, \omega\right)=$

$\sum_{n=-\infty}^{\infty}\left[E_{1 n}(\omega) J_{n}\left(k_{S_{l i n}} r_{1}\right)+F_{1 n}(\omega) Y_{n}\left(k_{S_{l i n}} r_{1}\right)\right] \mathrm{e}^{\mathrm{i} n \theta_{1}}$.

$Y_{n}$ is the cylindrical Bessel function of the second kind of integer order $n$, whereas $C_{1 n}(\omega), D_{1 n}(\omega), E_{1 n}(\omega)$, and $F_{1 n}(\omega)$ are uncertain refraction coefficients to be obtained.

Considering the boundary conditions, by assuming the no-slip condition between the tunnel lining and the rock medium, there is a continuity of the radial and shear stresses $\left(\sigma_{r r}\right.$ and $\left.\sigma_{r \theta}\right)$ and radial and shear displacements $\left(u_{r}\right.$ and $\left.u_{\theta}\right)$ along the lining's outer surface $\left(r_{1}=b_{1}\right.$, $\left.0 \leq \theta_{1} \leq 2 \pi\right): \sigma_{r r 1, \text { med }}=\sigma_{r r 1, \text { lin }}, \sigma_{r \theta 1, \text { med }}=\sigma_{r \theta 1, \text { lin }}, u_{r 1, \text { med }}=u_{r 1, \text { lin }}$, and $u_{\theta 1, \text { med }}=u_{\theta 1, \text { lin }}$. Along the inner surface of the lining $\left(r_{1}=a_{1}, 0 \leq \theta_{1} \leq 2 \pi\right)$, the radial and shear stress components are equal to zero: $\sigma_{r r 1, \text { lin }}=0$ and $\sigma_{r \theta 1, \text { lin }}=0$. The latter boundary conditions also apply to the cavity contour $\left(r_{2}=b_{2}, 0 \leq \theta_{2} \leq 2 \pi\right): \sigma_{r r 2}=0$ and $\sigma_{r \theta 2}=0$.

Utilizing the expressions for stresses and displacements in terms of the displacement potentials and recurrence formulas for the derivatives of Bessel functions [25], the expressions for the relevant stresses and displacements are obtained, with the terms $R_{h l}^{(q)}$ (see Appendix, Eqs. (23)-(32)). The subscript $h=1, \ldots, 5$ stands for the relevant stress/displacement components (radial stress $\sigma_{r r}$, hoop (circumferential, tangential) stress $\sigma_{\theta \theta}$, shear stress $\sigma_{r \theta}$, radial displacement $u_{r}$, and shear displacement $u_{\theta}$, respectively), $l=1,2$ for the corresponding potential ( $\varphi$ and $\psi$, respectively), whereas $\mathfrak{C}_{n}^{(q)}(q=1,2,3)$ is used to identify Bessel functions $\left(J_{n}, Y_{n}\right.$, and $H_{n}^{(1)}$, respectively).

By substitution of the expressions for stresses and displacements into the given boundary conditions along the outer and inner lining surfaces and the cavity contour, a linear system of equations of infinite order $n$ is obtained, with the unknown scattering and refraction coefficients:

$$
\begin{aligned}
& \tilde{\mu}\left[\vartheta_{1 n} \mathcal{R}_{12}^{(1)}\left(k_{S_{\text {med }}} b_{1}\right)+A_{1 n} \mathcal{R}_{11}^{(3)}\left(k_{P_{\text {med }}} b_{1}\right)+B_{1 n} \mathcal{R}_{12}^{(3)}\left(k_{S_{\text {med }}} b_{1}\right)\right. \\
& +\sum_{m=-\infty}^{\infty} A_{2 m} H_{m-n}^{(1)}\left(k_{P_{\text {med }}} d\right) \mathcal{R}_{11}^{(1)}\left(k_{P_{\text {med }}} b_{1}\right) \mathrm{e}^{\mathrm{i}(m-n) \theta_{21}} \\
& +\sum_{m=-\infty}^{\infty} B_{2 m} H_{m-n}^{(1)}\left(k_{S_{\text {med }}} d\right) \mathcal{R}_{12}^{(1)}\left(k_{S_{\text {med }}} b_{1}\right) \mathrm{e}^{\left.\mathrm{i}(m-n) \theta_{21}\right]} \\
& -C_{1 n} \mathcal{R}_{11}^{(1)}\left(k_{P_{\text {lin }}} b_{1}\right)-D_{1 n} \mathcal{R}_{11}^{(2)}\left(k_{P_{\text {lin }}} b_{1}\right) \\
& -E_{1 n} \mathcal{R}_{12}^{(1)}\left(k_{S_{\text {lin }}} b_{1}\right)-F_{1 n} \mathcal{R}_{12}^{(2)}\left(k_{S_{\text {lin }}} b_{1}\right)=0, \\
& \tilde{\mu}\left[\vartheta_{1 n} \mathcal{R}_{32}^{(1)}\left(k_{S_{\text {med }}} b_{1}\right)+A_{1 n} \mathcal{R}_{31}^{(3)}\left(k_{P_{\text {med }}} b_{1}\right)+B_{1 n} \mathcal{R}_{32}^{(3)}\left(k_{S_{\text {med }}} b_{1}\right)\right. \\
& +\sum_{m=-\infty}^{\infty} A_{2 m} H_{m-n}^{(1)}\left(k_{P_{\text {med }}} d\right) \mathcal{R}_{31}^{(1)}\left(k_{P_{\text {med }}} b_{1}\right) \mathrm{e}^{\mathrm{i}(m-n) \theta_{21}} \\
& +\sum_{m=-\infty}^{\infty} B_{2 m} H_{m-n}^{(1)}\left(k_{S_{\text {med }}} d\right) \mathcal{R}_{32}^{(1)}\left(k_{S_{\text {med }}} b_{1}\right) \mathrm{e}^{\mathrm{i}(m-n) \theta_{21}} \\
& -C_{1 n} \mathcal{R}_{31}^{(1)}\left(k_{P_{\text {lin }}} b_{1}\right)-D_{1 n} \mathcal{R}_{31}^{(2)}\left(k_{P_{\text {lin }}} b_{1}\right) \\
& -E_{1 n} \mathcal{R}_{32}^{(1)}\left(k_{S_{\text {lin }}} b_{1}\right)-F_{1 n} \mathcal{R}_{32}^{(2)}\left(k_{S_{\text {lin }}} b_{1}\right)=0,
\end{aligned}
$$




$$
\begin{aligned}
& \vartheta_{1 n} \mathcal{R}_{42}^{(1)}\left(k_{S_{\text {med }}} b_{1}\right)+A_{1 n} \mathcal{R}_{41}^{(3)}\left(k_{P_{\text {med }}} b_{1}\right)+B_{1 n} \mathcal{R}_{42}^{(3)}\left(k_{S_{\text {med }}} b_{1}\right) \\
& +\sum_{m=-\infty}^{\infty} A_{2 m} H_{m-n}^{(1)}\left(k_{P_{\text {med }}} d\right) \mathcal{R}_{41}^{(1)}\left(k_{P_{\text {med }}} b_{1}\right) \mathrm{e}^{\mathrm{i}(m-n) \theta_{21}} \\
& +\sum_{m=-\infty}^{\infty} B_{2 m} H_{m-n}^{(1)}\left(k_{S_{\text {med }}} d\right) \mathcal{R}_{42}^{(1)}\left(k_{S_{\text {med }}} b_{1}\right) \mathrm{e}^{\mathrm{i}(m-n) \theta_{21}} \\
& -C_{1 n} \mathcal{R}_{41}^{(1)}\left(k_{P_{\text {lin }}} b_{1}\right)-D_{1 n} \mathcal{R}_{41}^{(2)} \\
& -E_{1 n} \mathcal{R}_{42}^{(1)}\left(k_{S_{l i n}} b_{1}\right)-F_{1 n} \mathcal{R}_{42}^{(2)}\left(k_{S_{l i n}} b_{1}\right)=0 \text {, } \\
& \vartheta_{1 n} \mathcal{R}_{52}^{(1)}\left(k_{S_{\text {med }}} b_{1}\right)+A_{1 n} \mathcal{R}_{51}^{(3)}\left(k_{P_{\text {med }}} b_{1}\right)+B_{1 n} \mathcal{R}_{52}^{(3)}\left(k_{S_{\text {med }}} b_{1}\right) \\
& +\sum_{m=-\infty}^{\infty} A_{2 m} H_{m-n}^{(1)}\left(k_{P_{\text {med }}} d\right) \mathcal{R}_{51}^{(1)}\left(k_{P_{\text {med }}} b_{1}\right) \mathrm{e}^{\mathrm{i}(m-n) \theta_{21}} \\
& +\sum_{m=-\infty}^{\infty} B_{2 m} H_{m-n}^{(1)}\left(k_{S_{\text {med }}} d\right) \mathcal{R}_{52}^{(1)}\left(k_{S_{\text {med }}} b_{1}\right) \mathrm{e}^{\mathrm{i}(m-n) \theta_{21}} \\
& -C_{1 n} \mathcal{R}_{51}^{(1)}\left(k_{P_{\text {lin }}} b_{1}\right)-D_{1 n} \mathcal{R}_{51}^{(2)}\left(k_{P_{\text {lin }}} b_{1}\right) \\
& -E_{1 n} \mathcal{R}_{52}^{(1)}\left(k_{S_{l i n}} b_{1}\right)-F_{1 n} \mathcal{R}_{52}^{(2)}\left(k_{S_{l i n}} b_{1}\right)=0, \\
& C_{1 n} \mathcal{R}_{11}^{(1)}\left(k_{P_{\text {lin }}} a_{1}\right)+D_{1 n} \mathcal{R}_{11}^{(2)}\left(k_{P_{\text {lin }}} a_{1}\right) \\
& +E_{1 n} \mathcal{R}_{12}^{(1)}\left(k_{S_{l i n}} a_{1}\right)+F_{1 n} \mathcal{R}_{12}^{(2)}\left(k_{S_{l i n}} a_{1}\right)=0, \\
& C_{1 n} \mathcal{R}_{31}^{(1)}\left(k_{P_{i n}} a_{1}\right)+D_{1 n} \mathcal{R}_{31}^{(2)}\left(k_{P_{i n}} a_{1}\right) \\
& +E_{1 n} \mathcal{R}_{32}^{(1)}\left(k_{S_{l i n}} a_{1}\right)+F_{1 n} \mathcal{R}_{32}^{(2)}\left(k_{S_{l i n}} a_{1}\right)=0 \text {, }
\end{aligned}
$$$$
\vartheta_{2 n} \mathcal{R}_{12}^{(1)}\left(k_{S_{\text {med }}} b_{2}\right)+A_{2 n} \mathcal{R}_{11}^{(3)}\left(k_{P_{\text {med }}} b_{2}\right)+B_{2 n} \mathcal{R}_{12}^{(3)}\left(k_{S_{\text {med }}} b_{2}\right)
$$$$
+\sum_{m=-\infty}^{\infty} A_{1 m} H_{m-n}^{(1)}\left(k_{P_{m e d}} d\right) \mathcal{R}_{11}^{(1)}\left(k_{P_{\text {med }}} b_{2}\right) \mathrm{e}^{\mathrm{i}(m-n) \theta_{12}}
$$$$
+\sum_{m=-\infty}^{\infty} B_{1 m} H_{m-n}^{(1)}\left(k_{S_{\text {med }}} d\right) \mathcal{R}_{12}^{(1)}\left(k_{S_{\text {med }}} b_{2}\right) \mathrm{e}^{\mathrm{i}(m-n) \theta_{12}}=0,
$$$$
\vartheta_{2 n} \mathcal{R}_{32}^{(1)}\left(k_{S_{\text {med }}} b_{2}\right)+A_{2 n} \mathcal{R}_{31}^{(3)}\left(k_{P_{\text {med }}} b_{2}\right)+B_{2 n} \mathcal{R}_{32}^{(3)}\left(k_{S_{\text {med }}} b_{2}\right)
$$$$
+\sum_{m=-\infty}^{\infty} A_{1 m} H_{m-n}^{(1)}\left(k_{P_{\text {med }}} d\right) \mathcal{R}_{31}^{(1)}\left(k_{P_{\text {med }}} b_{2}\right) \mathrm{e}^{\mathrm{i}(m-n) \theta_{12}}
$$$$
+\sum_{m=-\infty}^{\infty} B_{1 m} H_{m-n}^{(1)}\left(k_{S_{m e d}} d\right) \mathcal{R}_{32}^{(1)}\left(k_{S_{m e d}} b_{2}\right) \mathrm{e}^{\mathrm{i}(m-n) \theta_{12}}=0 .
$$

In Eqs. (14-21), by the ratio of the shear modulus of the medium to that of the lining $\left(\tilde{\mu}=\mu_{\text {med }} / \mu_{\text {lin }}\right)$, the interaction of the tunnel and the surrounding ground is taken into consideration, as a highly significant phenomenon associated with tunnels [27, 28]. For solving the problem based on the derived theoretical solution, a program is developed by using the software Matlab ${ }^{\circledR}$.

\section{Convergence of the derived solution}

With an aim to test the convergence of infinite FourierBessel series of the derived solution, the dynamic stress concentration factor (DSCF) [25] at the inner surface of the lining $\left(r_{1}=a_{1}\right)$ and at the cavity contour $\left(r_{2}=b_{2}\right)$ of the tunnel-cavity system with the truncation of number $n$ is firstly evaluated. DSCF is the ratio of the relevant stress in the vicinity or along the boundary of an underground structure to the effective stress of the incident wave at the same point in a medium with no opening. For the hoop stress, which is the only one of all stress components that exists at the inner surface of a tunnel and at a cavity contour, DSCF $=\left|\sigma_{\theta \theta}^{*}\right|=\left|\sigma_{\theta \theta} / \tau_{0}\right|\left(\tau_{0}=\mu_{\text {med }} k_{\text {Smed }}^{2} \psi_{0}\right.$ is the stress amplitude of an incident SV-wave).

The geometric properties of the tunnel are $b_{1}=5.0 \mathrm{~m}$ and $b_{1} / a_{1}=1.1$, and of the cavity $b_{2}=5.0 \mathrm{~m}\left(=b_{1}\right)$. The material properties of the rock mass and the tunnel lining are: $\lambda_{\text {med }}=9.184 \cdot 10^{9} \mathrm{~N} / \mathrm{m}^{2}, \mu_{\text {med }}=3.936 \cdot 10^{9} \mathrm{~N} / \mathrm{m}^{2}$, $\rho_{\text {med }}=2242 \mathrm{~kg} / \mathrm{m}^{3} ; \lambda_{\text {lin }}=8.75 \cdot 10^{9} \mathrm{~N} / \mathrm{m}^{2}, \mu_{\text {lin }}=1.312 \cdot 10^{9} \mathrm{~N} / \mathrm{m}^{2}$, $\rho_{\text {lin }}=2548 \mathrm{~kg} / \mathrm{m}^{3}$. The minimum tunnel-to-cavity central distance considered in the study is set to be three times larger than the radius of the tunnel/cavity $\left(d / b_{1}=d / b_{2}=3\right)$, so that the width of the rock between the structures is equal to the size of their radius. The end-on seismic wave incidence $\left(\theta_{0}=0\right)$ upon the tunnel-cavity system has been considered in the analyses, since it is a highly important in-plane incidence case for two closely located tunnels aligned side-by-side [18, 19] (that is, the new-bored tunnel cavity is closely positioned behind the existing tunnel, i.e., the cavity is "in the shadow" of the tunnel). In addition, the high frequency incidence case (dimensionless wavenumber $k_{\text {Smed }} b_{1} \geq 3.0$ ) is considered in the convergence tests, as the series solution converges much more slowly for shorter incident wavelengths [25]. According to the performed analysis, the number of the series members $(n=m)$ that guarantees the accuracy of the calculation and the convergence of the solution is set to be 30 (Fig. 2(a)). 
Since the following analyses will also consider the region around the existing tunnel structure at the site of the planned cavity, the convergence tests for distances from the single tunnel greater than its radius are also carried out for an incident SV-wave of a high frequency. As presented in Fig. 2(b), the series solution converges much more slowly for greater distances from the tunnel. Hence, to ensure the convergence of the solution for this particular case under consideration, the series number $(n)$ is set to be 60 .

\section{Parametric study and analysis of results}

Unlike the cases of two cavities and two tunnels, when two structures are constructed simultaneously and when DSCF is a relevant indicator of the modification of the primary dynamic stress state in the ground, in the considered case of construction of a new tunnel next to the existing one, the secondary stress field should be taken into account. Thus, a new dynamic stress alteration factor $\left|\sigma_{\theta \theta}^{* *}\right|$ is introduced as a reasonable indicator for considering the effect of the presence of a new bored neighboring cavity on the seismic response of an existing tunnel and its surrounding medium. It represents the ratio of the seismically induced hoop stress for a tunnel-cavity system $\left(\left|\sigma_{\theta \theta}\right|^{\mathrm{TC}}\right)$ and the hoop stress for a single tunnel $\left(\left|\sigma_{\theta \theta}\right|^{\mathrm{ST}}\right)$, where two asterisks are used to designate the secondary stress field:

$\left|\sigma_{\theta \theta}^{* *}\right|=\left|\sigma_{\theta \theta}\right|^{\mathrm{TC}} /\left|\sigma_{\theta \theta}\right|^{\mathrm{ST}}$.

The effect of the space between the tunnel and the cavity on the dynamic response of an existing lined tunnel and its surrounding medium due to the presence of a newly driven unlined cavity is investigated by the proposed solution, for the end-on SV-wave incidence upon the tunnel-cavity system, at selected frequencies $k_{\text {Smed }} b_{1}=0.1,1.0$, and 3.0 (a low, intermediate, and high excitation frequency, respectively).

The dynamic response of the tunnel for various tunnel-cavity central distances $\left(d=3 b_{1}, 5 b_{1}\right.$, and $\left.10 b_{1}\right)$ is displayed in Fig. 3. From the polar distribution of the hoop stress amplitudes at the inner lining surface, it can be observed that at the low excitation frequency, as the distance between the tunnel and the cavity is increased to $d=10 b_{1}$, the DSCF values considering the tunnel-cavity system match the values typical for the single-tunnel case (Fig. 3(c)). The values of the dynamic stress alteration factor $\left|\sigma_{\theta \theta}^{* *}\right|$ match almost perfectly with a circle corresponding to a value of 1.0 (Fig. 3(f)) (exceptions are the sections at $\pm \pi / 2$, as a factor value slightly higher than 1.0 is conditioned by significantly low hoop stress values for the single-tunnel case). This implies that at the low incidence

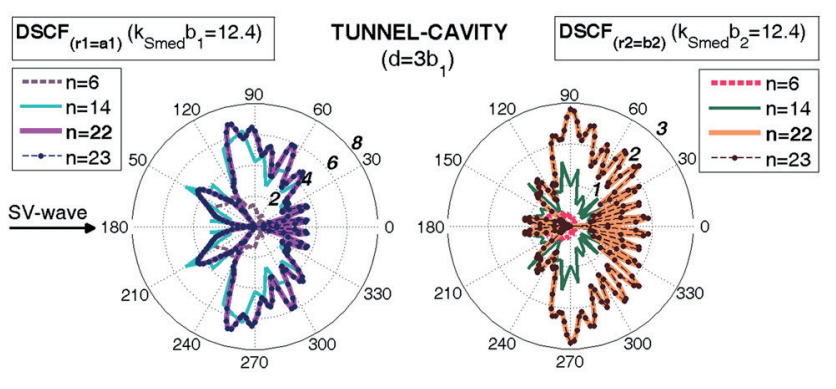

(a)
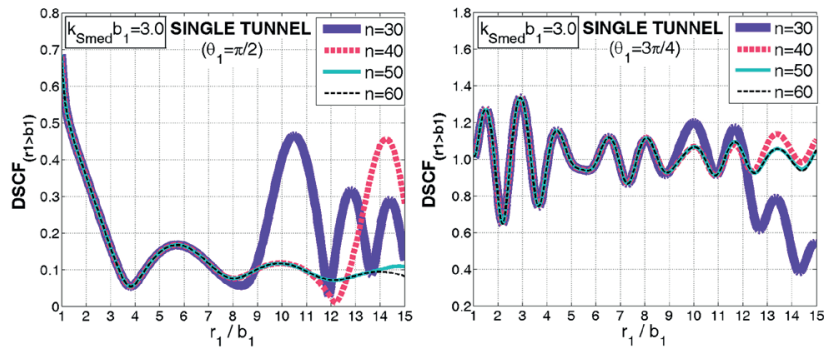

(b)

Fig. 2 Convergence test: (a) angular distribution of DSCF along the inner surface of the lining $\left(r_{1}=a_{1}\right)$ and the cavity contour $\left(r_{2}=b_{2}\right)$ of the tunnel-cavity system; (b) distribution of DSCF for increasing distance from a single tunnel $\left(r_{1}>b_{1}\right)$ at the considered sections

frequency, at the space ratio between the two neighboring structures of $d / b_{1}=10$, the multiple scattering and interaction effects may safely be neglected as the tunnel responds to a seismic excitation as a single one. At intermediate and high wavenumbers, for the given tunnel-cavity distance, the $\left|\sigma_{\theta \theta}^{* *}\right|$ factor curves oscillate around the value of 1.0 (Figs. 3(1),(r)), which points to the effect of the cavity on the adjacent tunnel from the standpoint of multi-scattering effects. Yet, this effect is slightly attenuated when compared to that at smaller distances (Figs. 3(j)-(k); (p)-(q)).

The analyses next examine the effect of the presence of a new bored neighboring cavity on the seismic response of the surrounding medium behind an existing tunnel (Fig. 4). For the low frequency incidence, an enormous increase of DSCF along diagonal planes of the cavity cross-section from the diagrams presented in Figs. 4(a)-(c) is evident very clearly when compared to DSCF in the ground behind the tunnel for the single-tunnel case. Furthermore, at the distance from the tunnel of $d=10 b_{1}$, the DSCF patterns considering the tunnel-cavity system and the single-cavity case almost perfectly match (Fig. 4(c)). Therefore, as previously inferred considering the tunnel, the insignificance of the interaction effects at this distance for the low frequency incidence was also confirmed considering the cavity. At intermediate and high excitation frequencies, for the considered distance of $d=10 b_{1}$, the 

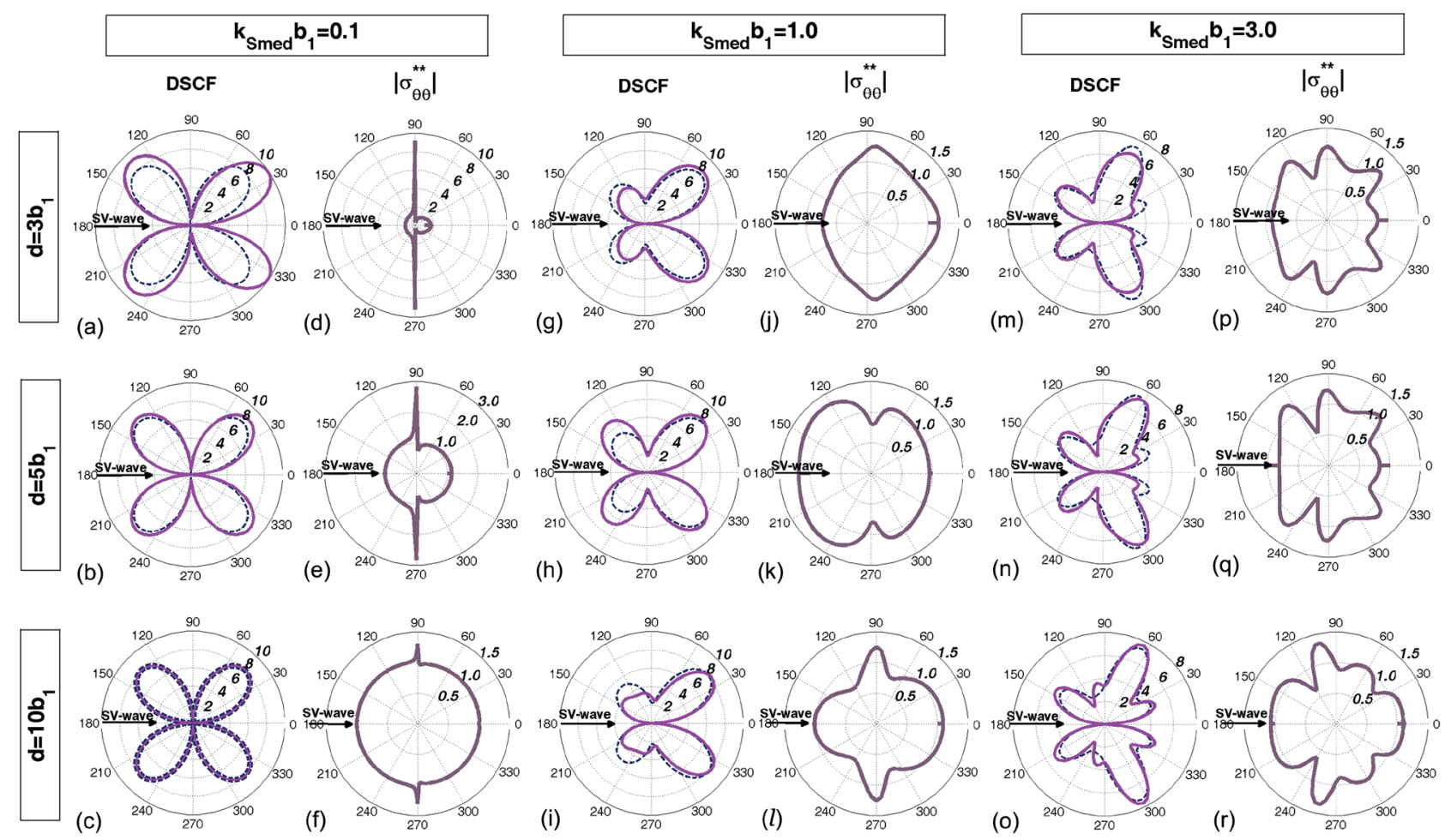

LEGEND $\quad---\mathrm{DSCF}_{(\mathrm{r} 1=\mathrm{a} 1)}\left(\right.$ single tunnel) $\quad-\mathrm{DSCF}_{(\mathrm{r} 1=\mathrm{a} 1)}$ (tunnel-cavity) $\quad-\left|\sigma_{\theta \theta 1, \operatorname{lin}}^{* *}\right|_{(\mathrm{r} 1=\mathrm{a} 1)}$ (tunnel-cavity)

Fig. 3 Polar distribution of DSCF and $\left|\sigma_{\theta \theta}^{* *}\right|$ at the inner lining surface for selected frequencies with increasing tunnel-cavity distance $\left(d=3 b_{1}, 5 b_{1}, 10 b_{1}\right)$
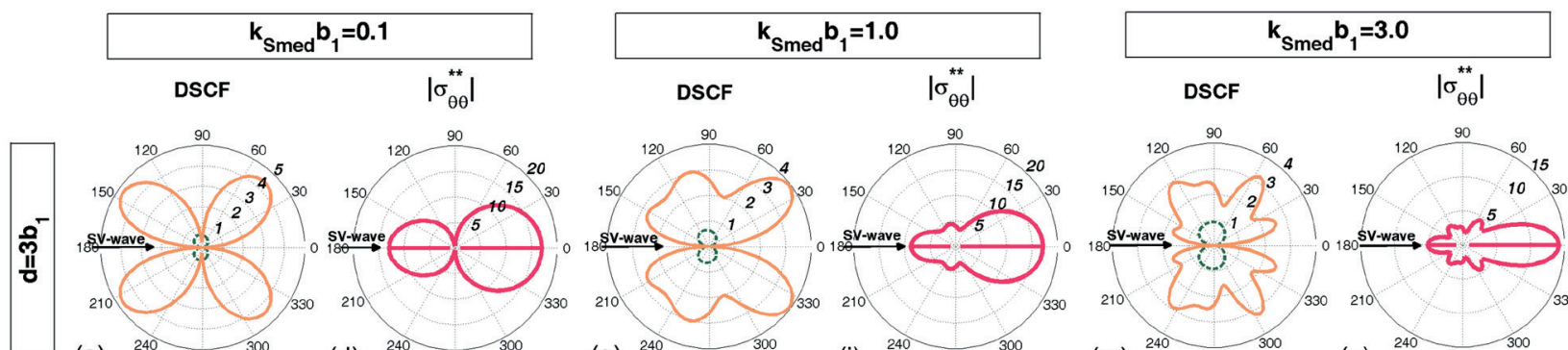

(a)

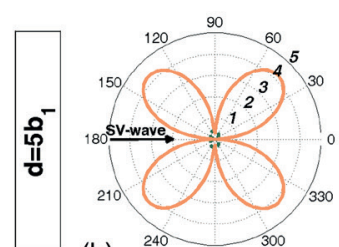

(d)
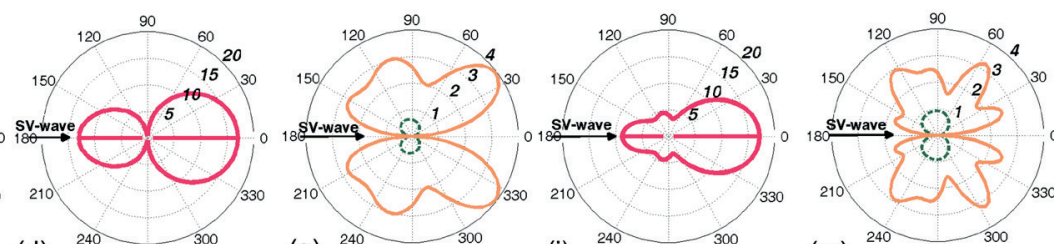

(m) $240-\frac{}{270} 300$
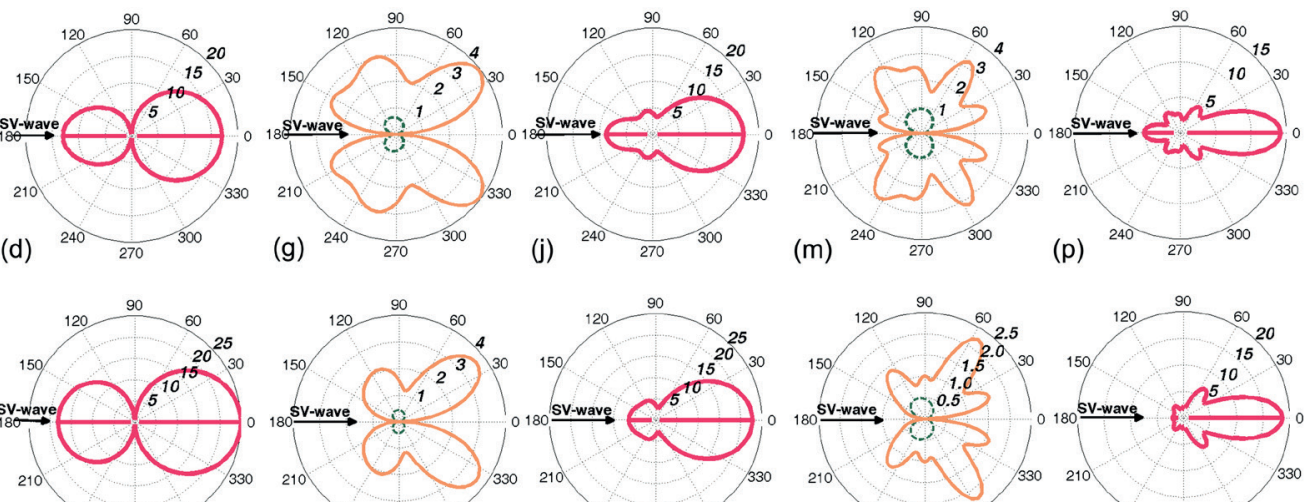

(g) $\begin{array}{lll}240 & 270 & \text { (j) } 240-270\end{array}$

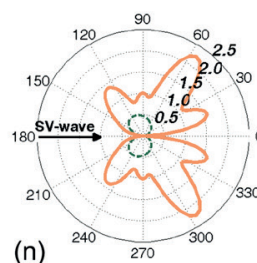

(p) $240-270$

(b) $240 \quad 270 \quad 300$

(e)
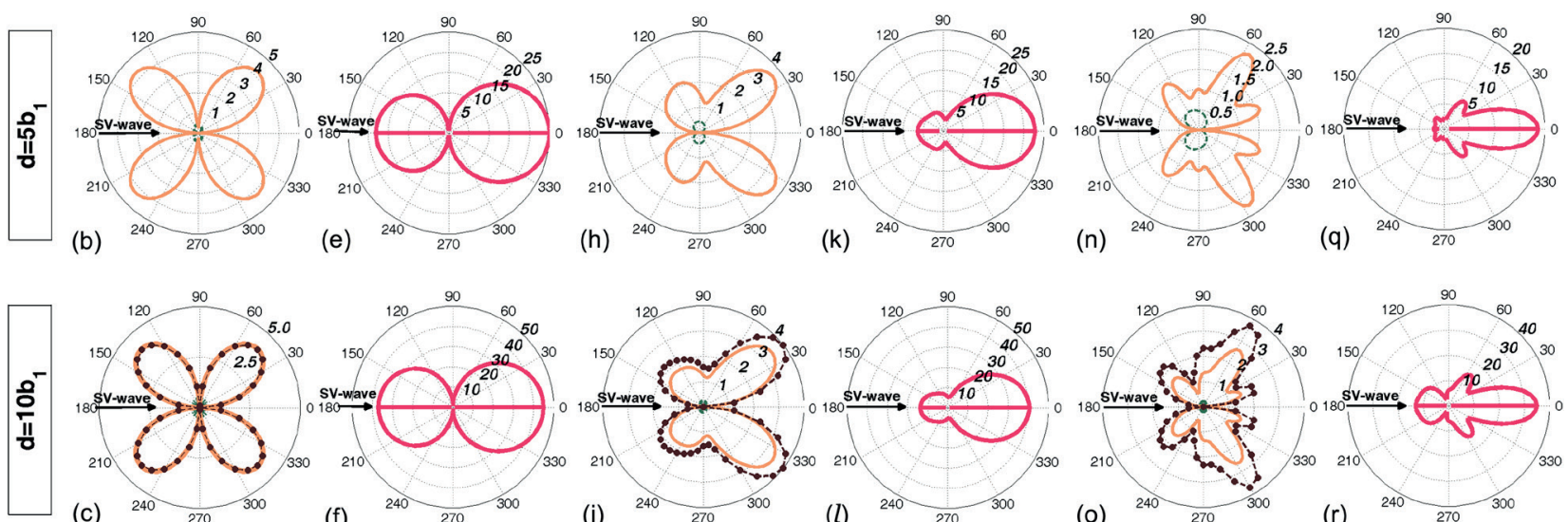

(k) ${ }^{240} \underset{270}{300}$

(n) $240-270 \quad 300$

(q) $240 \quad 270 \quad 300$

(c) $240-270$

(f) $240-270$

(i)
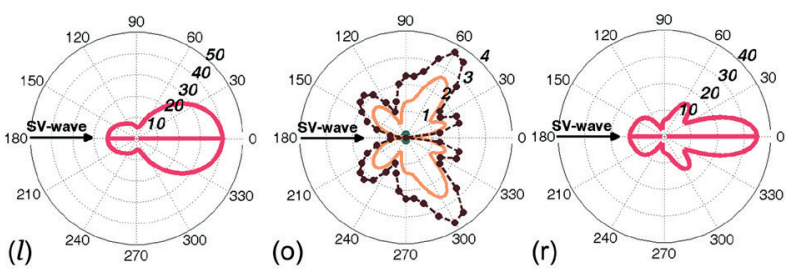

(r) $240-270 \quad 300$

LEGEND -.--DSCF $F_{(r 1>b 1 \rightarrow r 2=b 2)}$ (single tunnel) $\quad-\mathrm{DSCF}_{(\mathrm{r} 2=\mathrm{b} 2)}$ (tunnel-cavity) $\quad \ldots-\cdots \mathrm{DSCF}_{(\mathrm{r} 2=\mathrm{b} 2)}$ (single cavity) $\quad-\left|\sigma_{\theta \theta 2}^{* *}\right|_{(\mathrm{r} 2=\mathrm{b} 2)}$ (tunnel-cavity)

Fig. 4 Polar distribution of DSCF and $\left|\sigma_{\theta \theta}^{* *}\right|$ at the cavity contour for selected frequencies with increasing tunnel-cavity distance $\left(d=3 b_{1}, 5 b_{1}, 10 b_{1}\right)$ 
distribution forms of the DSCF curves at the cavity contour in the tunnel-cavity configuration and in the single-cavity case can be seen approaching (Figs. 4(i) and (o)). At all the selected frequencies, with increasing distance between the tunnel and the cavity, the peak values of the dynamic stress alteration factor along the cavity contour are also increased, being remarkably multiplied (Figs. 4(d)-(f); (j)-(1); (p)-(r)), in sections around the horizontal plane (the direction of an SV-wave propagation), as a result of the hoop stress amplitude drop in the ground behind the tunnel with increasing distance from the tunnel for the single-tunnel case.

The tunnel-cavity system, which is considered in the developed solution, in most cases could be considered a temporary configuration that occurs at the time of construction of a new (still unsupported) tunnel. However, the question logically arises as to whether the minimum seismically safe distance determined in the event of an earthquake on the tunnel-cavity system would still be appropriate in a similar incident after the completion of a new tunnel that is expected to be supported during the operational stage. Bearing in mind that in that case a part of the seismic energy would be additionally transmitted through the lining of the newly built tunnel, this may lead to the conclusion that the seismic waves, which are scattered through the rock mass between the two lined tunnels, could be considered slightly weakened. This could, accordingly, indicate that the minimum seismically safe distance, established for the former case (ten radii of the tunnel, i.e., five diameters of the tunnel), could be considered sufficiently safe for the latter case as well. Considering a single tunnel structure, it has been proven that in case of a stiffer liner in soft rock medium, an increase of the lining thickness yields the reduction of the dynamic stress concentration factor in the medium, as well as the decreasing of the stress in the lining [25].

When considering the minimum seismically safe distance between twin-tunnels, there is a rather modest volume of research on this issue. What is known in this sense so far is that, for the case of deeply embedded twin unsupported tunnels (as the most unfavorable case, when both openings are unsupported) and the coinciding affection by compressional and shear waves (as the worst possible case) of arbitrary direction in the plane of the cross-section of two circular tunnels, in terms of the stability of the rock pillar between the two tunnels in the sense that it does not exceed the ultimate rock strength, the minimum seismically safe distance between the centers of the cross-sections of parallel tunnels of about four tunnel diameters is established [29]. This is a slightly lower value than that determined by the results of our study. In addition, it was found that the presence of a lining as a whole has a small effect on the load being carried by the rock pillar in-between two tunnel structures, i.e. an increase of the lining thickness of the tunnel to provide preservation of the rock pillar is not as efficient as would be expected.

All the aforementioned findings, however, should certainly be checked by a possible upgrade of the developed solution for the tunnel-cavity system for the case when the newly built tunnel is supported by a lining.

\section{Conclusions}

The present study sheds light on another significant aspect of the problems in elastodynamics that hasn't been extensively investigated so far, considering the influence of a newly-constructed neighboring cavity on the seismic response of an existing tunnel and surrounding medium associated with the multiple scattering and interaction effects. For this purpose, a new dynamic stress alteration factor is introduced, which represents the ratio of the seismically induced tangential stress for a tunnel-cavity system to the circumferential stress of a single tunnel.

The most significant observation is that the distance of five tunnel diameters could be considered a threshold that provides insignificance of the seismically induced interaction effects between a tunnel and a neighboring cavity, especially at lower incidence frequencies. Minimal distances between two parallel horizontally aligned tunnels, recommended in the literature from the static aspect, based on the results of this study, do not meet the safety requirements regarding the earthquake-induced multiple scattering and interaction effects between two structures in close proximity.

Nevertheless, there is a need for further investigations on a variety of cases, such as various incident angles of seismic waves and/or various relations between the ground stiffness and the tunnel lining stiffness.

The solution derived in this study is of closed form obtained for a specific case of a tunnel-cavity system, by establishing a constitutive model of material behavior, identifying boundary conditions, and combining them with equilibrium and compatibility equations. The solution is exact in the theoretical sense, but on one hand, it still represents an approximation of the real problem, since it is necessary to make assumptions on geometry, applied boundary conditions, and constitutive behavior in order to idealize the real physical problem and present it in a relatively simple and 
comprehensible mathematical form. Nevertheless, on the other hand, analytical methods provide exact solutions that, with greater accuracy, continue to be of the utmost importance in studying the physical nature of the problem. They represent the best criteria for checking other approximate solutions, as well as for verifying the accuracy of softwares based on numerical methods for modeling the problems.

The presented solution with respect to the tunnel-cavity system is derived for the case of circular-cylindrical tunnels, horizontally aligned and deeply embedded in an elastic, homogeneous, infinite medium, with a lining-ground interface of perfectly tied behavior, under the plane-strain conditions. The solution enables the consideration of a tunnel and a cavity with different diameter ratios, having in mind that the adjacent cavity can be built for different purposes. It also allows the seismic waves to be considered for a variety of in-plane incidence angles (e.g., diagonally or vertically propagating seismic waves), as well as for different layouts of neighboring tunnels (e.g., vertical or inclined alignment).

On the other hand, the derived solution can also be upgraded for a number of other particular cases. In addition to the hoop stress, the remaining stress components may also be taken into consideration. In particular, the amplification factor of shear stress on the interface between lining and surrounding rock would be of great importance to study. The interface region between the lining and the ground could be considered as partial slip or full-slip, particularly relevant for the case of high frequency excitation,

\section{References}

[1] Zlatanović, E., Bonić, Z., Davidović, N. "Contemporary Approaches to Natural Disaster Risk Management in Geotechnics", In: Gocić, M., Aronica, G.T., Stavroulakis, G. E., Trajković, S. (eds.) Natural Risk Management and Engineering, Springer, Cham, Switzerland, 2020, pp. 115-141.

https://doi.org/10.1007/978-3-030-39391-5_6

[2] Dobashi, H., Hatsuku, T., Ichimura, T., Hori, M., Yamada, T., Ohbo, N., Moriguchi, M., Itami, H. "Full 3D seismic response analysis of underground ramp tunnel structure using large-scale numerical computation", presented at Proceedings of the 14th World Conference on Earthquake Engineering, Beijing, China, Oct. 12-17, 2008.

[3] Yang, J. S., Yan, L., Deng, S. J., Li, G. L. "Interactions of four tunnels driven in squeezing fault zone of Wushaoling Tunnel", Tunnelling and Underground Space Technology, 21(3-4), p. 359, 2006. https://doi.org/10.1016/j.tust.2005.12.176

[4] Yamaguchi, I., Yamazaki, I., Kiritani, Y. "Study of ground-tunnel interactions of four shield tunnels driven in close proximity, in relation to design and construction of parallel shield tunnels", Tunnelling and Underground Space Technology, 13(3), pp. 289-304, 1998. https://doi.org/10.1016/S0886-7798(98)00063-7 as well as for shallow-laid tunnels. For the case of shallow-buried structures, the presence of the ground surface should be taken into account (additional reflected waves in the half-space). For the case of poroelastic medium, the additional scattered Biot slow compressional wave, due to displacements of the solid frame with respect to pore water, must not be overlooked, pore fluid pressure has to be taken into account, and additional specific boundary conditions in terms of the interface permeability should be employed. In order to consider a degraded or disturbed zone, typical for the rock medium in real tunneling conditions, an attempt could be made toward implementing complex frequency dependent constants, thus accounting for the material damping and attenuation. The proposed solution can be extended to a three-dimensional 3D dynamic solution by considering the additional scattered field potential associated with the horizontal component of a shear wave (SH-wave). In addition, 3D analysis enables an obliquely incident seismic wave, with an arbitrary out-of-plane incidence angle, to be accounted for. Given the geometry of tunnels, it should be said that the applied method of wave function expansion is suitable for structures of regular shape, except for circular ones, and for cylinders of elliptical or parabolic cross-section.

\section{Acknowledgement}

The authors gratefully acknowledge the support of the Ministry of Education, Science, and Technological Development of the Republic of Serbia (Project TR36028).

[5] Li, W., Chen, Q. "Seismic damage evaluation of an entire underground subway system in dense urban areas by 3D FE simulation", Tunnelling and Underground Space Technology, 99, Article number: $103351,2020$.

https://doi.org/10.1016/j.tust.2020.103351

[6] Zlatanovic, E., Sesov, V., Lukic, D., Bonic, Z., Davidovic, N. "Seismic Design Codes for Tunnels and Underground Structures", Scientific Journal of Civil Engineering, 8(2), pp. 49-54, 2019. [online] Available at: http://gf.ukim.edu.mk/wp-content/uploads/ 2016/11/Vol.-8_Issue-2.pdf

[7] Zitron, N. R. "Multiple Scattering of Elastic Waves by Two Arbitrary Cylinders", Journal of the Acoustical Society of America, 42, pp. 620-624, 1967.

https://doi.org/10.1121/1.1910633

[8] Varadan, V. K., Varadan, V. V., Pao, Y.-H. "Multiple scattering of elastic waves by cylinders of arbitrary cross section: I SH waves", Journal of Acoustical Society of America, 63, pp. 1310-1319, 1978. https://doi.org/10.1121/1.381883 
[9] Providakis, C. P., Sotiropoulos, D. A., Beskos, D. E. "BEM analysis of reduced dynamic stress-concentration by multiple holes", Communications in Numerical Methods in Engineering, 9(11), pp. 917-924, 1993. https://doi.org/10.1002/cnm.1640091108

[10] Liang, J., Zhang, H., Lee, V. W. "A series solution for surface motion amplification due to underground twin tunnels: Incident SV waves", Earthquake Engineering and Engineering Vibration, 2(2), pp. 289298, 2003.

https://doi.org/10.1007/s11803-003-0012-x

[11] Liu, Q., Wang, R. "Dynamic response of twin closely-spaced circular tunnels to harmonic plane waves in a full space", Tunnelling and Underground Space Technology, 32, pp. 212-220, 2012. https://doi.org/10.1016/j.tust.2012.07.001

[12] Fotieva, N. N. "Calculation of the linings of two closely spaced circular tunnels for seismic effects", Soil Mechanics and Foundation Engineering, 15, pp. 186-193, 1978. https://doi.org/10.1007/BF02132798

[13] Moore, I. D., Guan, F. "Three-dimensional dynamic response of lined tunnels due to incident seismic waves", Earthquake Engineering and Structural Dynamics, 25(4), pp. 357-369, 1996.

https://doi.org/10.1002/(SICI)1096-9845(199604)25:4<357::AIDEQE556>3.0.CO;2-D

[14] Parvanova, S. L., Dineva, P. S., Manolis, G. D., Wuttke, F. "Seismic response of lined tunnels in the half-plane with surface topography", Bulletin of Earthquake Engineering, 12, pp. 981-1005, 2014. https://doi.org/10.1007/s10518-013-9546-0

[15] Lin, S.-Y., Hung, H.-H., Yang, J. P., Yang, Y. B. "Seismic Analysis of Twin Tunnels by a Finite/Infinite Element Approach", International Journal of Geomechanics, 17(9), pp. 1-16, 2017. https://doi.org/10.1061/(ASCE)GM.1943-5622.0000940

[16] Lu, Y., Huang, W. "Numerical Simulation of Dynamic Response Law of Intersecting Metro Tunnels in Upper and Lower Strata", Geotechnical and Geological Engineering, 38(5), pp. 3773-3785, 2020.

https://doi.org/10.1007/s10706-020-01257-x

[17] Wang, J. H., Zhou, X. L., Lu, J. F. "Dynamic stress concentration around elliptic cavities in saturated poroelastic soil under harmonic plane waves", International Journal of Solids and Structures, 42(14), pp. 4295-4310, 2005.

https://doi.org/10.1016/j.ijsolstr.2005.01.003

[18] Hasheminejad, S. M., Avazmohammadi, R. "Harmonic wave diffraction by two circular cavities in a poroelastic formation", Soil Dynamics and Earthquake Engineering, 27(1), pp. 29-41, 2007. https://doi.org/10.1016/j.soildyn.2006.05.001
[19] Hasheminejad, S. M., Avazmohammadi, R. "Dynamic Stress Concentrations in Lined Twin Tunnels within Fluid-Saturated Soil", Journal of Engineering Mechanics, 134(7), pp. 542-554, 2008. https://doi.org/10.1061/(ASCE)0733-9399(2008)134:7(542)

[20] Zhou, X.-L., Wang, J.-H., Jiang, L.-F. "Dynamic response of a pair of elliptic tunnels embedded in a poroelastic medium", Journal of Sound and Vibration, 325(4-5), pp. 816-834, 2009. https://doi.org/10.1016/j.jsv.2009.04.001

[21] Kim, S. H. "Interaction behaviours between parallel tunnels in soft ground", Tunnelling and Underground Space Technology, 19, p. 448, 2004.

https://doi.org/10.1016/j.tust.2004.02.058

[22] Hefny, A. M., Chua, H. C., Zhao, J. "Parametric studies on the interaction between existing and new bored tunnels", Tunnelling and Underground Space Technology, 19, p. 471, 2004.

https://doi.org/10.1016/j.tust.2004.02.074

[23] Dibavar, B. H., Ahmadi, M. H., Davarpanah, S. M. "3D Numerical Investigation of Ground Settlements Induced by Construction of Istanbul Twin Metro Tunnels with Special Focus on Tunnel Spacing", Periodica Polytechnica Civil Engineering, 63(4), pp. 1225-1234, 2019. https://doi.org/10.3311/PPci.14591

[24] Achenbach, J. D. "Wave propagation in elastic solids", North Holland Publishing Company, New York, NY, USA, 1973.

[25] Mow, C.-C., Pao, H.-Y. "The diffraction of elastic waves and dynamic stress concentrations", Rand, Santa Monica, CA, USA, Rep. R-482-PR, 1971.

[26] Ivanov, Y. A. "Diffraction of electromagnetic waves on two bodies", National Aeronautics and Space Administration, Washington, DC, USA, 1970.

[27] Zlatanović, E., Trajković-Milenković, M., Lukić, D. Č., Brčić, S., Šešov, V. "A comparison of linear and nonlinear seismic tunnelground interaction analyses", Acta Geotechnica Slovenica, 13(2), pp. 27-42, 2016. [online] Available at: http://www.fg.uni-mb.si/ journal-ags/2016-2/article-3.asp

[28] Liang, S., Chen, Z. "SPH-FEM coupled simulation of SSI for conducting seismic analysis on a rectangular underground structure", Bulletin of Earthquake Engineering, 17, pp. 159-180, 2019. https://oi.org/10.1007/s10518-018-0456-z

[29] Fotieva, N. N. "Determination of the minimum seismically safe distance between two parallel tunnels", Soil Mechanics and Foundation Engineering, 17, pp. 111-116, 1980. https://doi.org/10.1007/BF01710398 


\section{Appendix}

(23)

$\mathcal{R}_{11}^{(q)}\left(k_{P} r\right)=\left(n^{2}+n-\frac{k_{S}^{2} r^{2}}{2}\right) \mathfrak{C}_{n}^{(q)}\left(k_{P} r\right)-k_{P} r \mathfrak{C}_{n-1}^{(q)}\left(k_{P} r\right) \quad \mathcal{R}_{32}^{(q)}\left(k_{S} r\right)=-\left(n^{2}+n-\frac{k_{S}^{2} r^{2}}{2}\right) \mathfrak{C}_{n}^{(q)}\left(k_{S} r\right)+k_{S} r \mathfrak{C}_{n-1}^{(q)}\left(k_{S} r\right)$

$\mathcal{R}_{12}^{(q)}\left(k_{S} r\right)=\mathrm{in}\left[-(n+1) \mathfrak{C}_{n}^{(q)}\left(k_{S} r\right)+k_{S} r \mathfrak{C}_{n-1}^{(q)}\left(k_{S} r\right)\right]$

(24)

$\mathcal{R}_{41}^{(q)}\left(k_{P} r\right)=-n \mathfrak{C}_{n}^{(q)}\left(k_{P} r\right)+k_{P} r \mathfrak{C}_{n-1}^{(q)}\left(k_{P} r\right)$

$\mathcal{R}_{21}^{(q)}\left(k_{P} r\right)$

$\mathcal{R}_{42}^{(q)}\left(k_{S} r\right)=\mathrm{in} \mathfrak{C}_{n}^{(q)}\left(k_{S} r\right)$

$=-\left(n^{2}+n+\frac{k_{S}^{2} r^{2}}{2}-k_{P}^{2} r^{2}\right) \mathfrak{C}_{n}^{(q)}\left(k_{P} r\right)+k_{P} r \mathfrak{C}_{n-1}^{(q)}\left(k_{P} r\right)$

$\mathcal{R}_{22}^{(q)}\left(k_{S} r\right)=\mathrm{i} n\left[(n+1) \mathfrak{C}_{n}^{(q)}\left(k_{S} r\right)-k_{S} r \mathfrak{C}_{n-1}^{(q)}\left(k_{S} r\right)\right]$

$\mathcal{R}_{51}^{(q)}\left(k_{P} r\right)=\mathrm{in} \mathfrak{C}_{n}^{(q)}\left(k_{P} r\right)$

$\mathcal{R}_{31}^{(q)}\left(k_{P} r\right)=\mathrm{i} n\left[-(n+1) \mathfrak{C}_{n}^{(q)}\left(k_{P} r\right)+k_{P} r \mathfrak{C}_{n-1}^{(q)}\left(k_{P} r\right)\right]$ 